\title{
Temperature effects on optical properties and chemical composition of secondary organic aerosol derived from $n$-dodecane
}

\section{Junling Li et al.}

Correspondence to: Weigang Wang (wangwg@iccas.ac.cn)

The copyright of individual parts of the supplement might differ from the CC BY 4.0 License. 


\section{Estimation of the RI uncertainty}

The RI values are obtained with extinction efficiency $\left(Q_{e x t}\right)$ and surface mean diameter $\left(D_{s}\right)$. The uncertainty of the $D_{s}$ measured by SMPS is $\pm 1 \%$. The uncertainty of the extinction efficiency is calculated as the following:

The extinction efficiency can be expressed as:

$$
\mathrm{Q}_{\mathrm{ext}}=\alpha_{\mathrm{ext}} /\left(\frac{1}{4} \mathrm{~N} \pi \mathrm{D}^{2}\right)
$$

The three variables $\alpha_{\mathrm{ext}}, \mathrm{N}, \mathrm{D}$ were independent and the uncertainty of them are $3 \%, 10 \%$ and $1 \%$, respectively. Considering the propagation of uncertainty, we can obtain the variance formula:

$$
\sigma_{\mathrm{Q}}^{2}=\sigma_{\alpha}^{2}\left(\frac{\partial \mathrm{Q}}{\partial \alpha}\right)^{2}+\sigma_{\mathrm{N}}^{2}\left(\frac{\partial \mathrm{Q}}{\partial \mathrm{N}}\right)^{2}+\sigma_{\mathrm{D}}^{2}\left(\frac{\partial \mathrm{Q}}{\partial \mathrm{D}}\right)^{2}
$$

Then the uncertainty of $\mathrm{Q}_{\mathrm{ext}}$ can be calculated as:

$$
\begin{gathered}
\frac{\sigma_{\mathrm{Q}}}{\mathrm{Q}}=\sqrt{\left(\frac{\sigma_{\alpha}}{\alpha}\right)^{2}+\left(\frac{\sigma_{\mathrm{N}}}{\mathrm{N}}\right)^{2}+4\left(\frac{\sigma_{\mathrm{D}}}{\mathrm{D}}\right)^{2}}=\sqrt{0.03^{2}+0.1^{2}+4 \times 0.01^{2}} \\
=\sqrt{0.0113}=10.6 \%
\end{gathered}
$$

The measured extinction efficiency $\left(Q_{\text {ext,mea }}\right)$ is compared to calculated extinction efficiency $\left(Q_{e x t, c a l}\right)$. The best-fit RI value

40 is determined by minimizing the following reduced merit function $\left(\chi_{r}\right)$ :

$$
\chi_{r}=\frac{1}{N} \sum_{i=1}^{N}\left(Q_{\text {ext,mea }}-Q_{\text {ext }, \text { cal }}(n, k)\right)_{i}^{2}
$$

The uncertainty of the retrieval method is \pm 0.002 , and the uncertainty of the measured extinction efficiency is $\pm 10.6 \%$, resulting in the final uncertainty of the retrieved RI value to be about 0.02-0.03. 

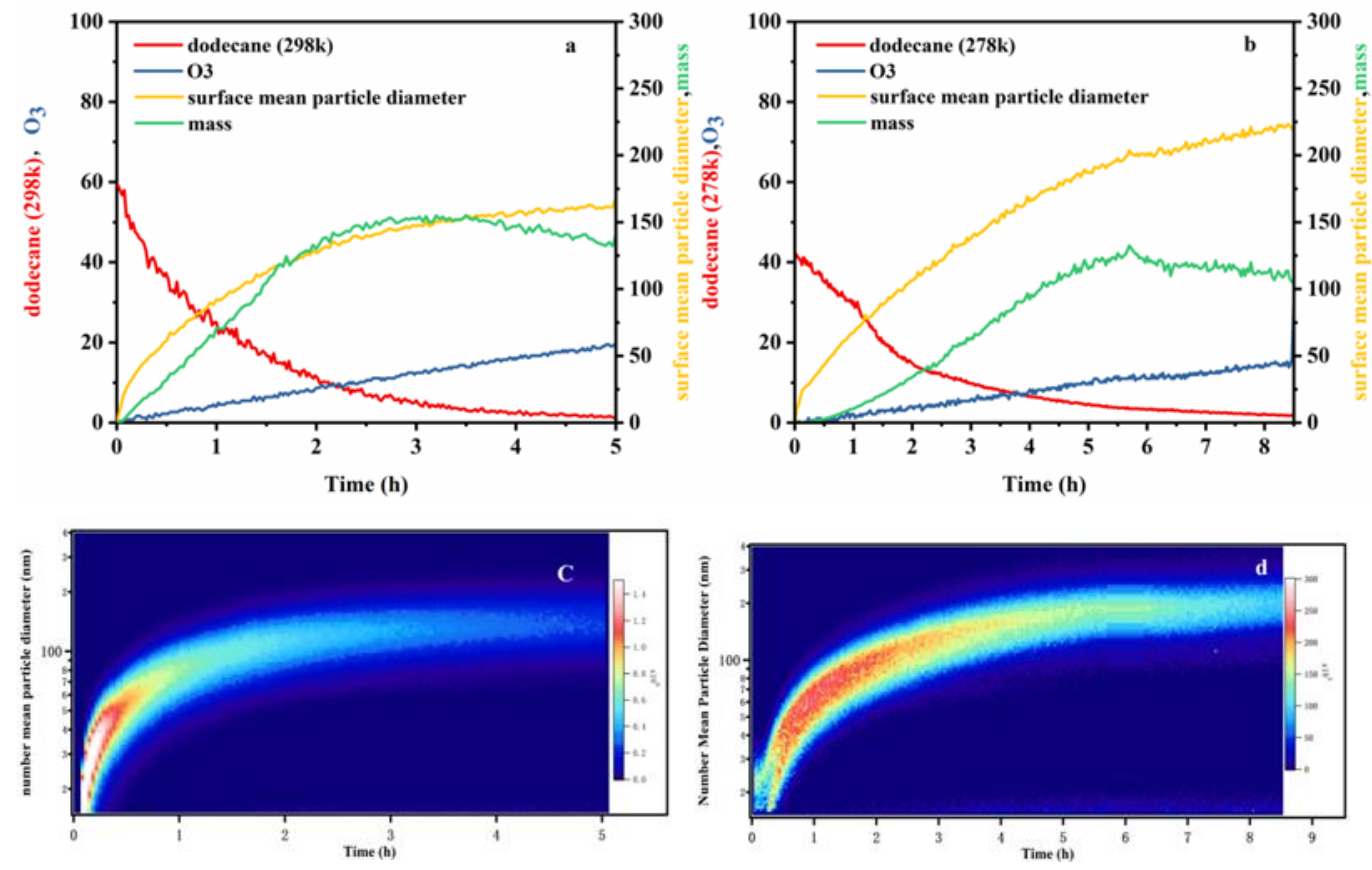

Figure S1: Reaction profiles of $\boldsymbol{n}$-dodecane photo-oxidation experiments at two different temperature conditions, (a) time-dependent concentration of dodecane, $\mathrm{O}_{3}$, surface mean particle diameter and aerosol mass (the density used in the system is $1.1 \mathrm{~g} / \mathrm{cm}^{3}$, measured with our CPMA and SMPS) at $25{ }^{\circ} \mathrm{C}$; (b) time-dependent concentration of dodecane, $\mathrm{O}_{3}$, surface mean particle diameter and aerosol mass (the density used in the system is $1.1 \mathrm{~g} / \mathrm{cm}^{3}$, measured with our CPMA and SMPS) at $5{ }^{\circ} \mathrm{C}$; (c) number size distribution of formed aerosol particles at $25{ }^{\circ} \mathrm{C}$; (d) number size distribution of formed aerosol particles at $5{ }^{\circ} \mathrm{C}$. 

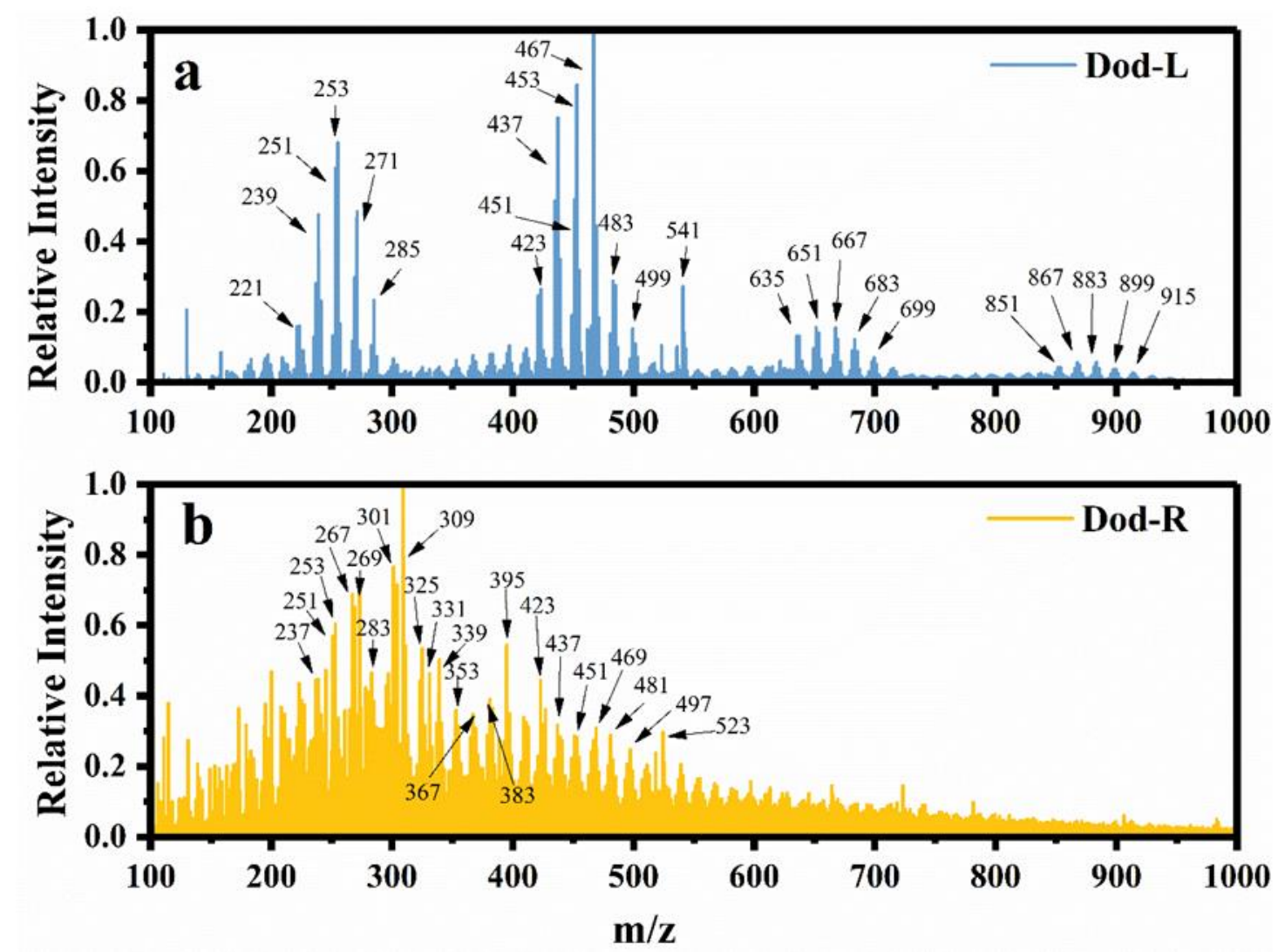

Figure S2: Mass specra of $\boldsymbol{n}$-dodecane SOAs obtained by ESI-TOF-MS in positive ion mode. (a) low temperature condition; (b) room temperature condition. 


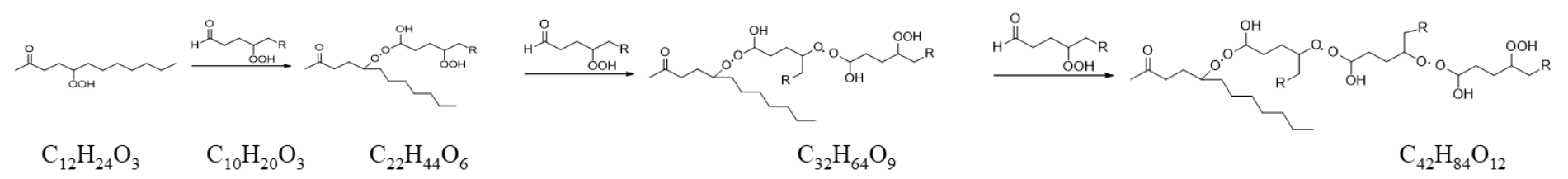

$\underbrace{\text { OOH }}_{\mathrm{C}_{12} \mathrm{H}_{26} \mathrm{O}_{3}}$

管 $\begin{array}{llll}\mathrm{C}_{12} \mathrm{H}_{24} \mathrm{O}_{4} & \mathrm{C}_{22} \mathrm{H}_{44} \mathrm{O}_{7} & \mathrm{C}_{32} \mathrm{H}_{64} \mathrm{O}_{10} & \mathrm{C}_{42} \mathrm{H}_{84} \mathrm{O}_{13}\end{array}$ 型 $\mathrm{C}_{12} \mathrm{H}_{22} \mathrm{O}_{4} \quad \mathrm{C}_{22} \mathrm{H}_{42} \mathrm{O}_{7} \quad \mathrm{C}_{32} \mathrm{H}_{62} \mathrm{O}_{10} \quad \mathrm{C}_{42} \mathrm{H}_{82} \mathrm{O}_{13}$ 每 $\mathrm{C}_{12} \mathrm{H}_{22} \mathrm{O}_{5} \quad \mathrm{C}_{22} \mathrm{H}_{42} \mathrm{O}_{8} \quad \mathrm{C}_{32} \mathrm{H}_{62} \mathrm{O}_{11} \quad \mathrm{C}_{42} \mathrm{H}_{82} \mathrm{O}_{14}$ $\underbrace{\text { OOH }}_{\text {OH }}$ $\mathrm{C}_{12} \mathrm{H}_{24} \mathrm{O}_{5} \quad \mathrm{C}_{22} \mathrm{H}_{44} \mathrm{O}_{8} \quad \mathrm{C}_{32} \mathrm{H}_{64} \mathrm{O}_{11} \quad \mathrm{C}_{42} \mathrm{H}_{84} \mathrm{O}_{14}$ $\underbrace{\text { OOH }}_{\mathrm{O}}$

$$
\mathrm{C}_{12} \mathrm{H}_{20} \mathrm{O}_{5} \quad \mathrm{C}_{22} \mathrm{H}_{40} \mathrm{O}_{8}
$$

$\mathrm{C}_{32} \mathrm{H}_{60} \mathrm{O}_{11}$ 


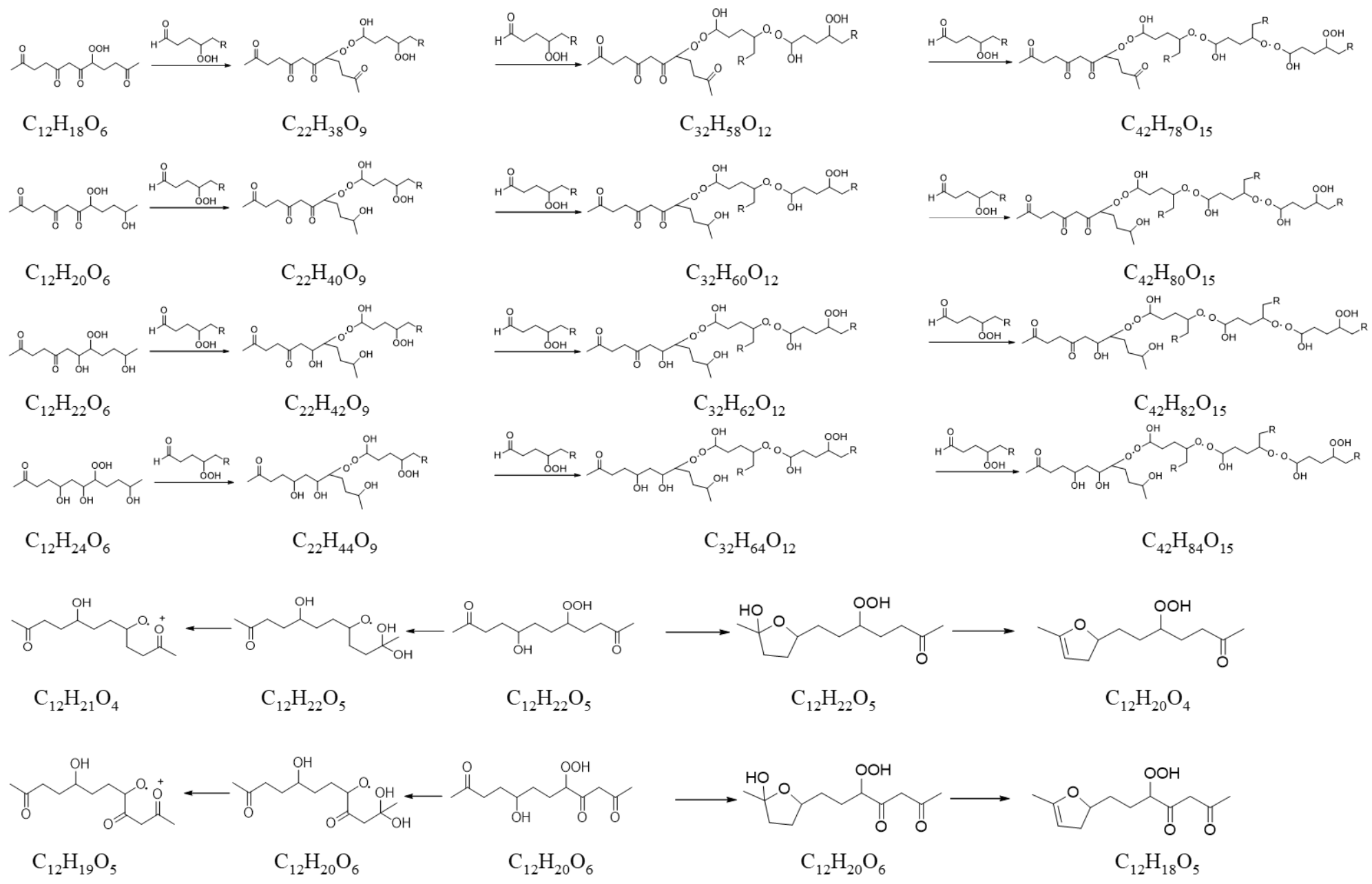

Figure S3: Scheme for forming 11 types of PHA oligomers from reaction of an aldehyde of carbon length 10, with various hydroperoxy compounds and scheme for forming two types of furan derivatives with two kind of hydroperoxy compounds. 

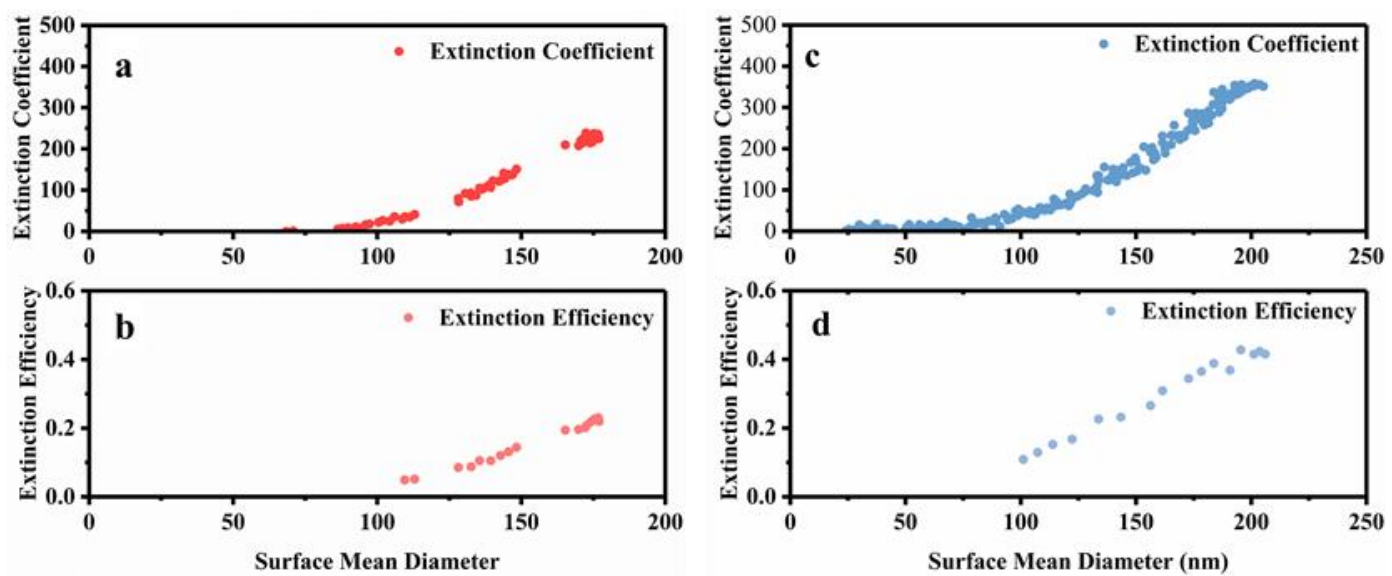

Figure S4: Evolution of optical parameters at $532 \mathrm{~nm}$. Extinction coefficient (a) and efficiency (b) at room temperature; extinction coefficient (c) and efficiency (d) at low temperature. 
Table S1: The calculated RI values for identified molecules under two temperature conditions. (Dod-R refers to the room temperature condition; Dod-L refers to the low temperature condition).

\begin{tabular}{|c|c|c|c|c|c|c|c|}
\hline Condition & Molecular Formula & $\begin{array}{c}\text { Molecular } \\
\text { Weight }\end{array}$ & $\mathbf{M}+\mathbf{H}$ & $\mathbf{M}+\mathbf{N a}$ & Polarizability & Unsaturation & $\begin{array}{c}\text { Predicted } \\
\text { RI }_{\text {cal }}\end{array}$ \\
\hline Dod-R & $\mathrm{C}_{10} \mathrm{H}_{12} \mathrm{O}_{3}$ & 180 & 181.08 & & 19.17 & 5 & 1.541 \\
\hline Dod-R & $\mathrm{C}_{10} \mathrm{H}_{14} \mathrm{O}_{3}$ & 182 & 183.10 & & 19.51 & 4 & 1.510 \\
\hline Dod-R & $\mathrm{C}_{10} \mathrm{H}_{16} \mathrm{O}_{3}$ & 184 & 185.117 & & 19.85 & 3 & 1.479 \\
\hline Dod-R & $\mathrm{C}_{10} \mathrm{H}_{20} \mathrm{O}_{2}$ & 172 & & 195.13 & 19.96 & 1 & 1.415 \\
\hline Dod-R & $\mathrm{C}_{8} \mathrm{H}_{14} \mathrm{O}_{4}$ & 174 & & 197.07 & 17.06 & 2 & 1.445 \\
\hline Dod-R & $\mathrm{C}_{12} \mathrm{H}_{22} \mathrm{O}_{2}$ & 198 & 199.17 & & 23.32 & 2 & 1.452 \\
\hline Dod-R & $\mathrm{C}_{10} \mathrm{H}_{16} \mathrm{O}_{4}$ & 200 & 201.11 & & 20.42 & 3 & 1.479 \\
\hline Dod-R & $\mathrm{C}_{8} \mathrm{H}_{12} \mathrm{O}_{6}$ & 204 & 205.17 & & 17.86 & 3 & 1.476 \\
\hline Dod-R & $\mathrm{C}_{11} \mathrm{H}_{12} \mathrm{O}_{4}$ & 208 & 209.08 & & 21.25 & 6 & 1.575 \\
\hline Dod-R & $\mathrm{C}_{10} \mathrm{H}_{20} \mathrm{O}_{3}$ & 188 & & 211.13 & 20.53 & 1 & 1.418 \\
\hline Dod-R & $\mathrm{C}_{8} \mathrm{H}_{14} \mathrm{O}_{5}$ & 190 & & 213.07 & 17.63 & 2 & 1.444 \\
\hline Dod-R & $\mathrm{C}_{11} \mathrm{H}_{18} \mathrm{O}_{4}$ & 214 & 215.12 & & 22.27 & 3 & 1.481 \\
\hline Dod-R & $\mathrm{C}_{10} \mathrm{H}_{16} \mathrm{O}_{5}$ & 216 & 217.11 & & 20.99 & 3 & 1.481 \\
\hline Dod-R & $\mathrm{C}_{12} \mathrm{H}_{20} \mathrm{O}_{2}$ & 196 & & 219.13 & 22.98 & 3 & 1.483 \\
\hline Dod-R & $\mathrm{C}_{12} \mathrm{H}_{22} \mathrm{O}_{2}$ & 198 & & 221.15 & 23.32 & 2 & 1.452 \\
\hline Dod-R & $\mathrm{C}_{10} \mathrm{H}_{16} \mathrm{O}_{4}$ & 200 & & 223.09 & 20.42 & 3 & 1.479 \\
\hline Dod-R & $\mathrm{C}_{10} \mathrm{H}_{18} \mathrm{O}_{4}$ & 224 & 225.11 & & 20.76 & 2 & 1.450 \\
\hline Dod-R & $\mathrm{C}_{12} \mathrm{H}_{18} \mathrm{O}_{4}$ & 226 & 227.13 & & 23.78 & 4 & 1.516 \\
\hline Dod-R & $\mathrm{C}_{10} \mathrm{H}_{12} \mathrm{O}_{6}$ & 228 & 229.07 & & 20.88 & 5 & 1.543 \\
\hline Dod-R & $\mathrm{C}_{11} \mathrm{H}_{14} \mathrm{O}_{4}$ & 210 & & 233.08 & 21.59 & 5 & 1.545 \\
\hline Dod-R & $\mathrm{C}_{12} \mathrm{H}_{20} \mathrm{O}_{3}$ & 212 & & 235.12 & 23.55 & 3 & 1.484 \\
\hline Dod-R & $\mathrm{C}_{12} \mathrm{H}_{22} \mathrm{O}_{3}$ & 214 & & 237.14 & 23.89 & 2 & 1.447 \\
\hline Dod-R & $\mathrm{C}_{12} \mathrm{H}_{24} \mathrm{O}_{3}$ & 216 & & 239.16 & 24.23 & 1 & 1.422 \\
\hline Dod-R & $\mathrm{C}_{10} \mathrm{H}_{18} \mathrm{O}_{5}$ & 218 & & 241.10 & 21.33 & 2 & 1.449 \\
\hline Dod-R & $\mathrm{C}_{10} \mathrm{H}_{20} \mathrm{O}_{5}$ & 220 & & 243.12 & 21.67 & 1 & 1.419 \\
\hline Dod-R & $\mathrm{C}_{9} \mathrm{H}_{18} \mathrm{O}_{6}$ & 222 & & 245.10 & 20.39 & 1 & 1.417 \\
\hline Dod-R & $\mathrm{C}_{11} \mathrm{H}_{18} \mathrm{O}_{6}$ & 246 & 247.11 & & 23.41 & 3 & 1.483 \\
\hline Dod-R & $\mathrm{C}_{12} \mathrm{H}_{18} \mathrm{O}_{4}$ & 226 & & 249.11 & 23.78 & 4 & 1.516 \\
\hline Dod-R & $\mathrm{C}_{11} \mathrm{H}_{22} \mathrm{O}_{6}$ & 250 & 251.15 & & 24.09 & 1 & 1.422 \\
\hline Dod-R & $\mathrm{C}_{10} \mathrm{H}_{20} \mathrm{O}_{7}$ & 252 & 253.13 & & 22.81 & 2 & 1.420 \\
\hline
\end{tabular}




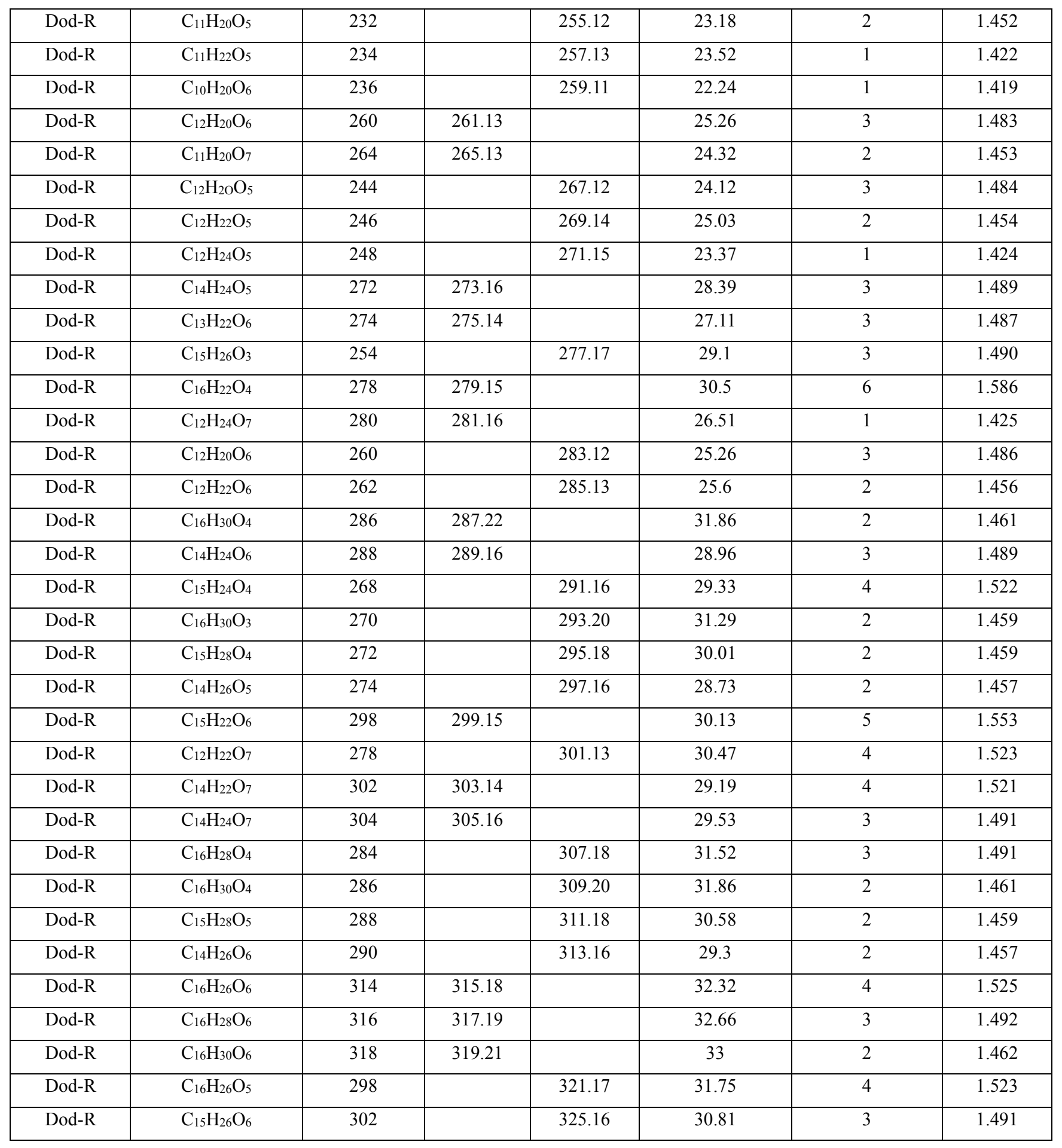




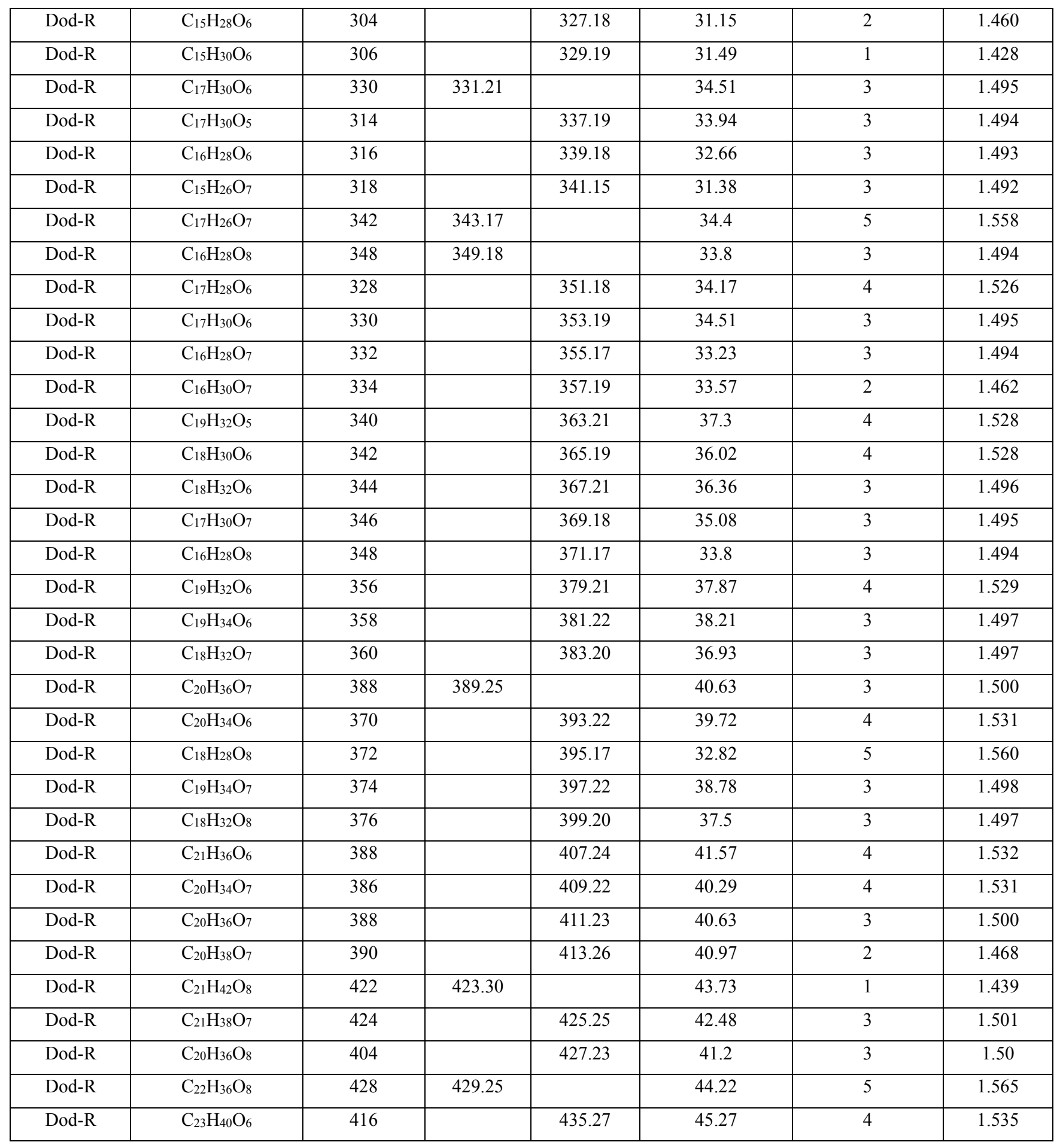




\begin{tabular}{|c|c|c|c|c|c|c|c|}
\hline Dod-R & $\mathrm{C}_{22} \mathrm{H}_{38} \mathrm{O}_{7}$ & 414 & & 437.25 & 43.99 & 4 & 1.534 \\
\hline Dod-R & $\mathrm{C}_{21} \mathrm{H}_{36} \mathrm{O}_{8}$ & 416 & & 439.23 & 42.71 & 4 & 1.532 \\
\hline Dod-R & $\mathrm{C}_{21} \mathrm{H}_{38} \mathrm{O}_{8}$ & 418 & & 441.24 & 43.05 & 3 & 1.501 \\
\hline Dod-R & $\mathrm{C}_{24} \mathrm{H}_{42} \mathrm{O}_{6}$ & 426 & & 449.28 & 47.12 & 4 & 1.536 \\
\hline Dod-R & $\mathrm{C}_{22} \mathrm{H}_{42} \mathrm{O}_{9}$ & 450 & 451.29 & & 45.81 & 2 & 1.472 \\
\hline Dod-R & $\mathrm{C}_{22} \mathrm{H}_{38} \mathrm{O}_{9}$ & 446 & & 469.24 & 45.13 & 4 & 1.534 \\
\hline Dod-R & $\mathrm{C}_{22} \mathrm{H}_{40} \mathrm{O}_{9}$ & 448 & & 471.26 & 45.47 & 3 & 1.502 \\
\hline Dod-R & $\mathrm{C}_{24} \mathrm{H}_{40} \mathrm{O}_{8}$ & 456 & & 479.26 & 47.92 & 5 & 1.568 \\
\hline Dod-R & $\mathrm{C}_{24} \mathrm{H}_{42} \mathrm{O}_{9}$ & 474 & & 497.27 & 48.83 & 4 & 1.537 \\
\hline Dod-R & $\mathrm{C}_{22} \mathrm{H}_{42} \mathrm{O}_{12}$ & 498 & 499.27 & & 47.52 & 2 & 1.473 \\
\hline Dod-R & $\mathrm{C}_{25} \mathrm{H}_{42} \mathrm{O}_{9}$ & 486 & & 509.27 & 50.34 & 5 & 1.570 \\
\hline Dod-R & $\mathrm{C}_{23} \mathrm{H}_{42} \mathrm{O}_{12}$ & 510 & 511.27 & & 49.03 & 3 & 1.506 \\
\hline Dod-R & $\mathrm{C}_{24} \mathrm{H}_{42} \mathrm{O}_{10}$ & 490 & & 513.26 & 49.4 & 4 & 1.538 \\
\hline Dod-R & $\mathrm{C}_{24} \mathrm{H}_{42} \mathrm{O}_{12}$ & 522 & 523.28 & & 50.54 & 4 & 1.539 \\
\hline Dod-R & $\mathrm{C}_{24} \mathrm{H}_{42} \mathrm{O}_{11}$ & 506 & & 529.26 & 49.97 & 4 & 1.538 \\
\hline Dod-L & $\mathrm{C}_{12} \mathrm{H}_{22} \mathrm{O}$ & 182 & 183.17 & & 22.75 & 2 & 1.451 \\
\hline Dod-L & $\mathrm{C}_{12} \mathrm{H}_{20} \mathrm{O}_{2}$ & 196 & 197.15 & & 22.98 & 3 & 1.483 \\
\hline Dod-L & $\mathrm{C}_{12} \mathrm{H}_{22} \mathrm{O}_{2}$ & 198 & 199.17 & & 23.32 & 2 & 1.452 \\
\hline Dod-L & $\mathrm{C}_{10} \mathrm{H}_{16} \mathrm{O}_{3}$ & 184 & & 207.10 & 19.85 & 3 & 1.479 \\
\hline Dod-L & $\mathrm{C}_{10} \mathrm{H}_{18} \mathrm{O}_{3}$ & 186 & & 209.11 & 20.19 & 2 & 1.447 \\
\hline Dod-L & $\mathrm{C}_{10} \mathrm{H}_{20} \mathrm{O}_{3}$ & 188 & & 211.13 & 20.53 & 1 & 1.415 \\
\hline Dod-L & $\mathrm{C}_{11} \mathrm{H}_{16} \mathrm{O}_{4}$ & 212 & 213.11 & & 21.93 & 4 & 1.514 \\
\hline Dod-L & $\mathrm{C}_{12} \mathrm{H}_{20} \mathrm{O}_{3}$ & 212 & & 235.13 & 23.55 & 3 & 1.484 \\
\hline
\end{tabular}




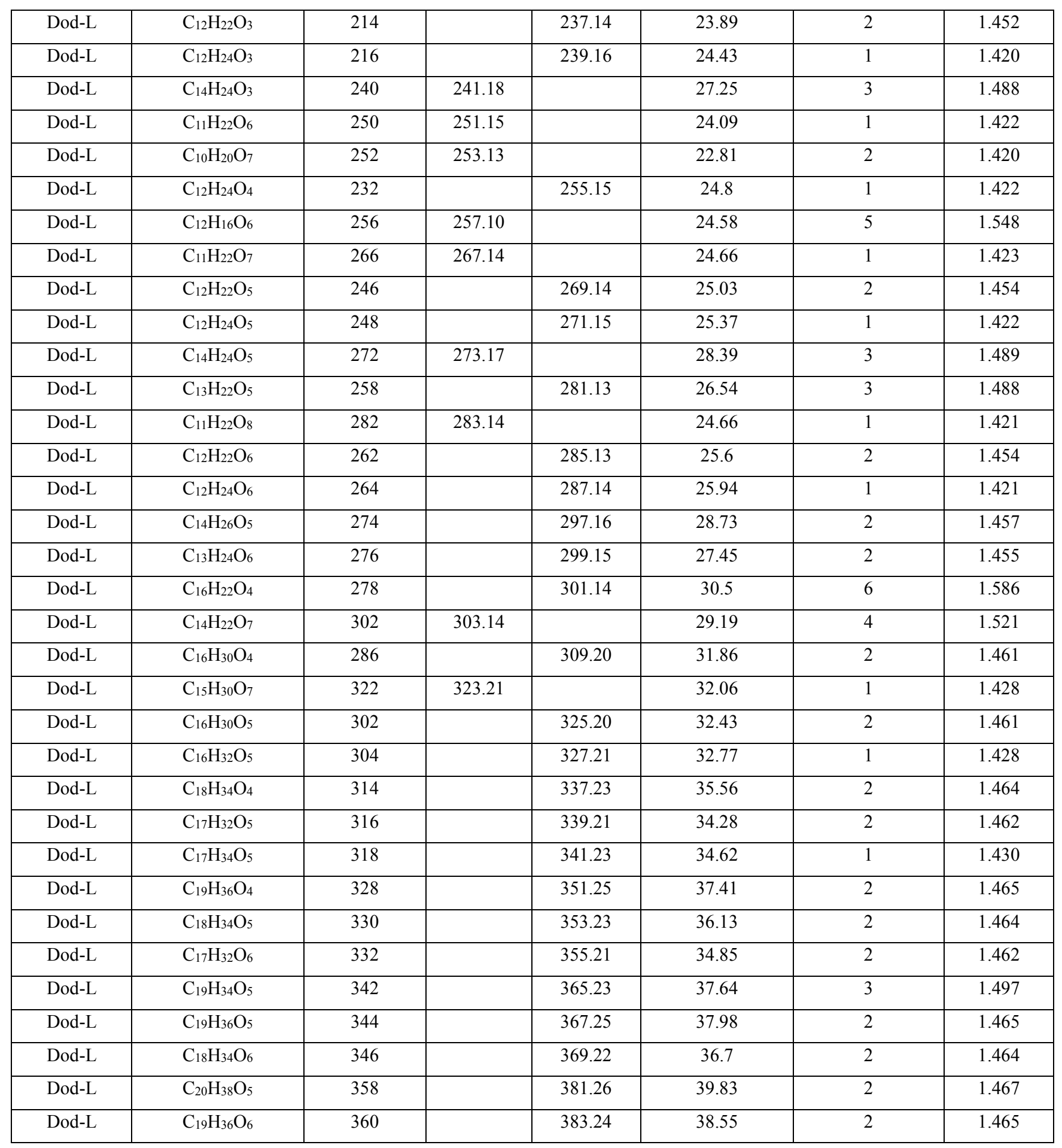




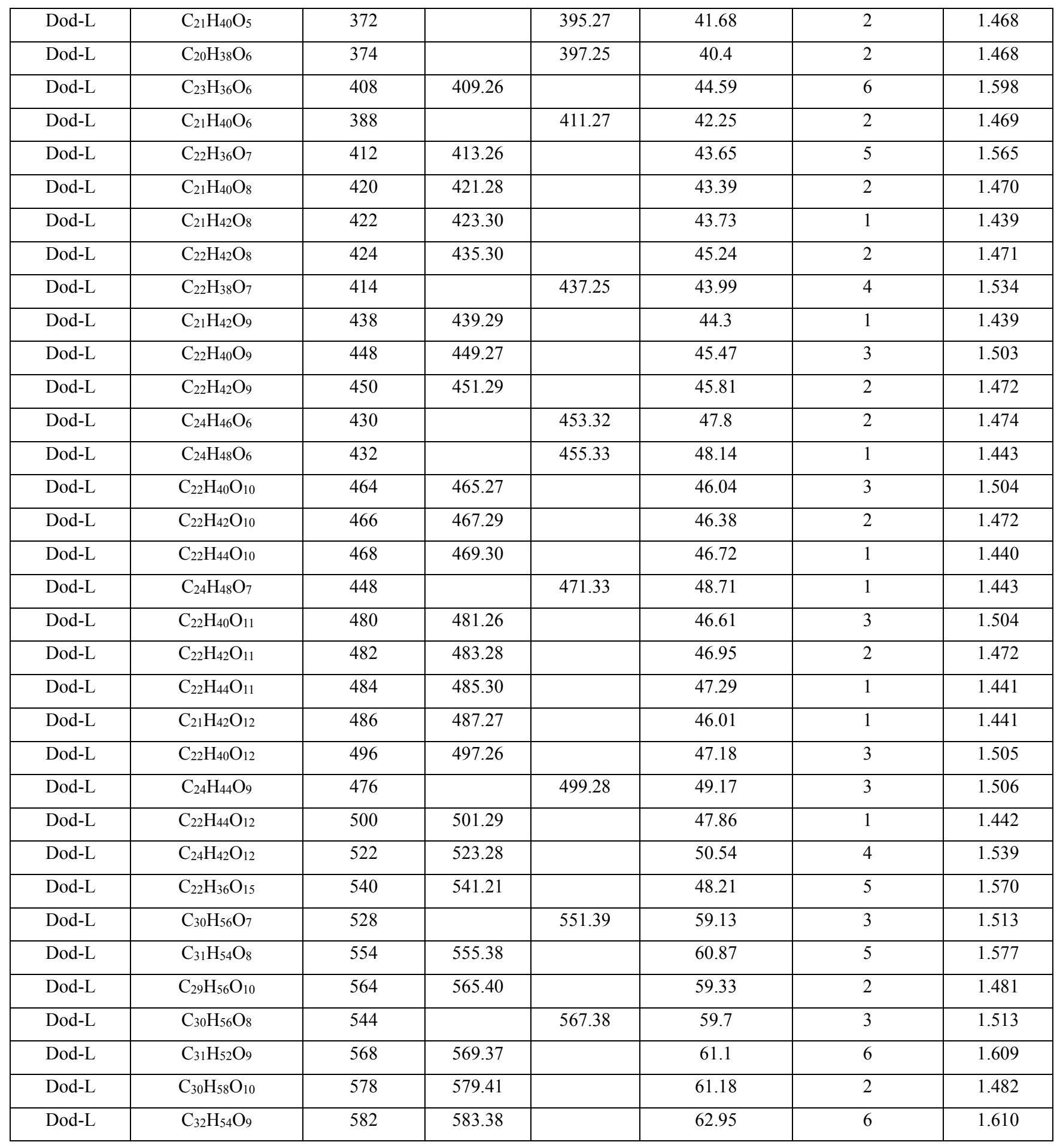




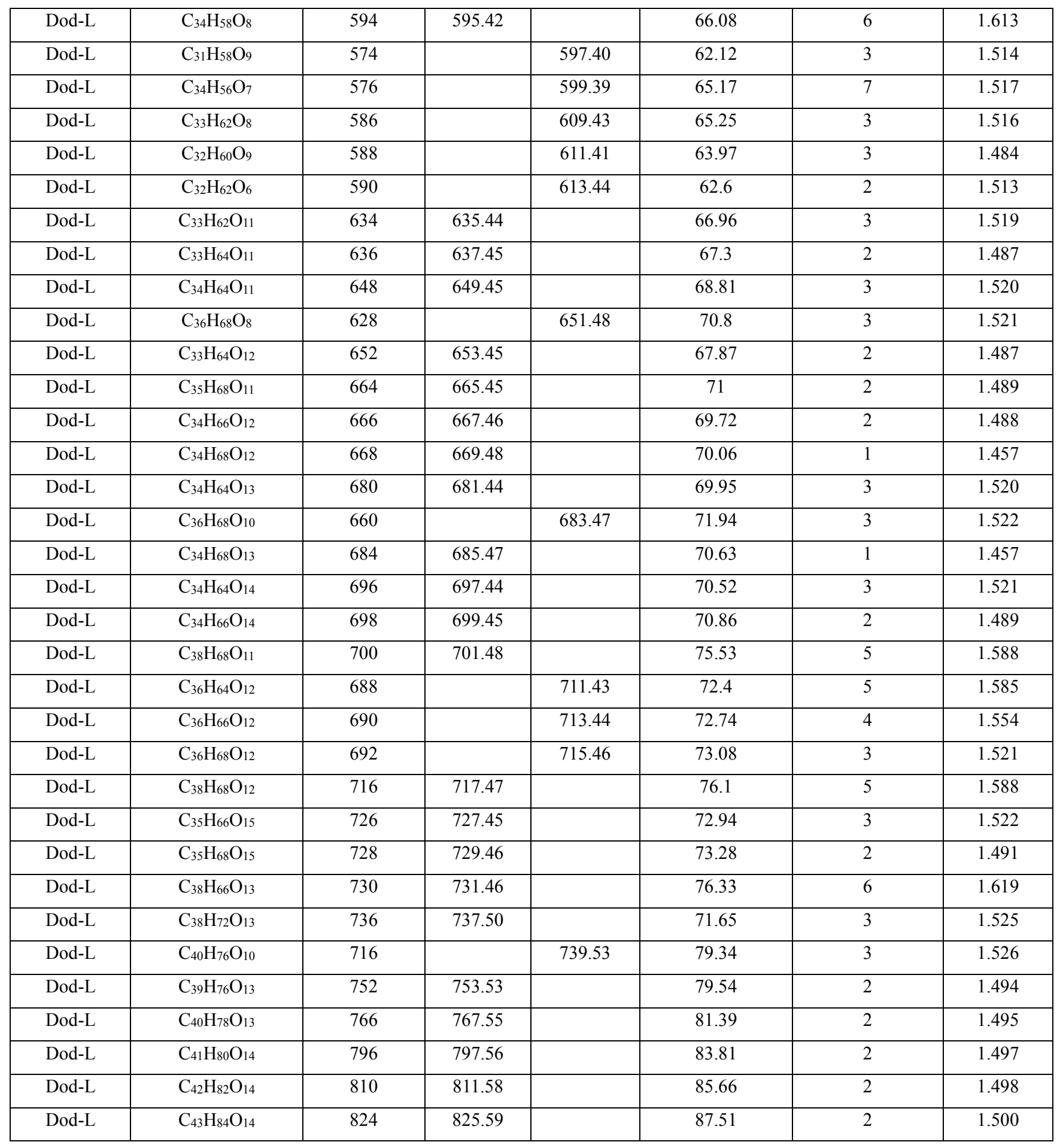




\begin{tabular}{|c|c|c|c|c|c|c|c|}
\hline Dod-L & $\mathrm{C}_{50} \mathrm{H}_{92} \mathrm{O}_{9}$ & 814 & & 837.68 & 96.59 & 5 & 1.601 \\
\hline Dod-L & $\mathrm{C}_{48} \mathrm{H}_{92} \mathrm{O}_{10}$ & 828 & & 851.65 & 94.14 & 3 & 1.536 \\
\hline Dod-L & $\mathrm{C}_{44} \mathrm{H}_{84} \mathrm{O}_{15}$ & 852 & 853.59 & & 89.59 & 3 & 1.533 \\
\hline Dod-L & $\mathrm{C}_{45} \mathrm{H}_{84} \mathrm{O}_{15}$ & 864 & 865.59 & & 91.1 & 4 & 1.566 \\
\hline Dod-L & $\mathrm{C}_{48} \mathrm{H}_{92} \mathrm{O}_{11}$ & 844 & & 867.65 & 94.71 & 3 & 1.536 \\
\hline Dod-L & $\mathrm{C}_{46} \mathrm{H}_{90} \mathrm{O}_{15}$ & 882 & 883.64 & & 93.63 & 2 & 1.503 \\
\hline Dod-L & $\mathrm{C}_{46} \mathrm{H}_{92} \mathrm{O}_{15}$ & 884 & 885.65 & & 93.97 & 1 & 1.472 \\
\hline Dod-L & $\mathrm{C}_{46} \mathrm{H}_{88} \mathrm{O}_{16}$ & 896 & 897.62 & & 93.86 & 3 & 1.536 \\
\hline
\end{tabular}

